\title{
A New Morphological Measure of Histogram Bimodality
}

\author{
Miguel Angel Cataño and Joan Climent \\ 1 Pontificia Universidad Católica del Perú \\ 2 Barcelona Tech (UPC), Spain
}

\begin{abstract}
The presence of multiple modes in a histogram gives important information about data distribution for a great amount of different applications. The dip test has been the most common statistical measure used for this purpose.

Histograms of oriented gradients (HOGs) with a high bimodality have shown to be very useful to detect highly robust keypoints. However, the dip test presents serious disadvantages when dealing with such histograms. In this paper we describe the drawbacks of the dip test for determining HOGs bimodality, and present a new bimodality test, based on mathematical morphology, that overcomes them.
\end{abstract}

Keywords: Keypoint detection, Bimodality test, Histograms of Oriented Gradients, Mathematical Morphology, Dynamics.

\section{Introduction}

Histograms of oriented gradients (HOGs) have become increasingly popular in the computer vision community in recent years. Among other applications, they are used for object detection [3], as keypoint descriptors (i.e., SIFT [8]), or for keypoint detection 2. The basic idea is that local shapes can be characterized rather well by the distribution of local gradient directions. The use of directions gives robustness to lighting changes. HOGs are implemented by dividing the image into small windows, called cells, each cell accumulating a 1-D histogram of gradient directions of all pixels within the cell. The main advantage of HOG representation is the high invariance to translations and rotations.

Recent works have shown that HOGs can be used for the detection of singular points, giving very robust keypoints. They use the bimodality of the HOGs as a measure of the significance of a keypoint. The dip test of bimodality [5] is used for this purpose. Figure 1 shows the HOGs corresponding to the cells selected in the image of figure $2 \mathrm{a}$. The bimodality of each histogram is computed for each pixel using the dip test. Figure $2 \mathrm{~b}$ shows the bimodality image extracted from figure 2a, As it can be seen, peaks of the bimodality image correspond to keypoints of the original image.

However, the dip measure has some drawbacks, mainly due to the fact that HOGs are cyclic, and the extension of the dip test to cyclic histograms is not 


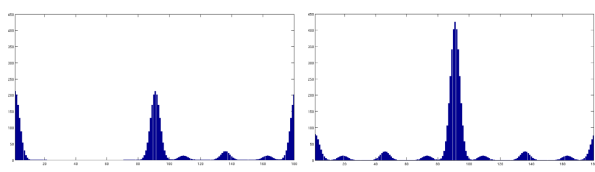

(a) cell $1 \mathrm{HOG}$

(b) cell $2 \mathrm{HOG}$

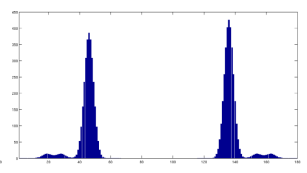

(c) cell $3 \mathrm{HOG}$

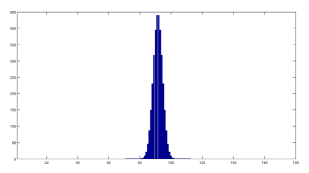

(d) cell 4 HOG

Fig. 1. HOGs of selected areas of figure 2a

trivial. Furthermore, it presents a remarkable lack of linearity, specially in the particular case where the histogram has samples only in two neighboring bins.

In this paper we present a new bimodality measure, based on the dynamics, that will be used to improve the detection of keypoints. The dynamics is a mathematical morphology concept introduced by Grimaud [4]. It has usually been used as a concise and powerful measure of contrast for the identification of regions of interest in the image, but in our work we use dynamics to quantify the modes of the histograms. The dynamics of the histogram peaks are used to define a new bimodality measure, that makes possible to establish a hierarchy of interest points.

After presenting the basics of the dip test and the dynamics in section 2, we give the implementation details of our proposal in section 3 . In the results section, the limitations of the dip measure are shown, and we compare its response to histogram transformations with the response of our method.

\section{Preliminaries}

\subsection{The Dip Measure}

The most popular measure of bimodality is the dip Test [5]. It was first used for globular cluster studies. The dip test is based on the cumulative distribution of the input sample. The dip statistic is the maximum distance between the cumulative input distribution and the best-fitting unimodal distribution. The dip test searches specifically for a flat step in the cumulative distribution function, which corresponds to a 'dip' in the histogram representation. Formally, the dip
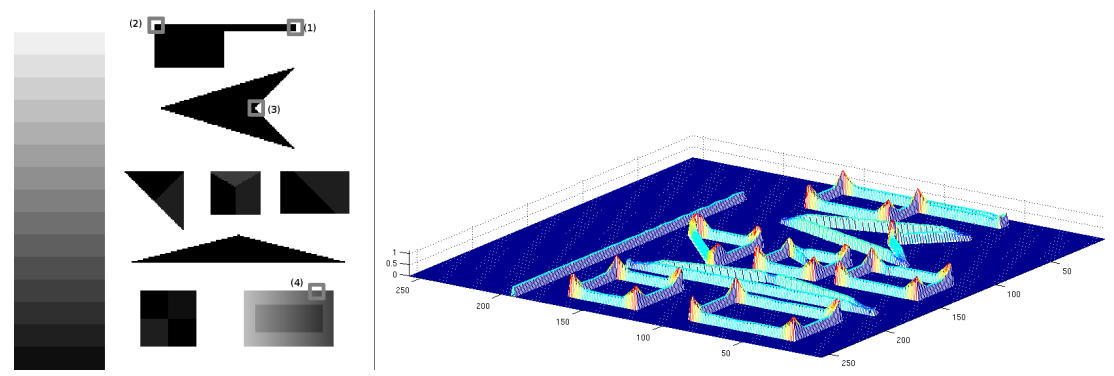

Fig. 2. (a) A synthetic image (b) A 3D representation of the bimodality image 
of a distribution function $F$ is defined by $D(F)=\rho(F, \zeta)$, being $\zeta$ the class of unimodal distribution functions, and

$$
\rho(F, \zeta)=\inf _{G \in \zeta}\left\{\sup _{x}|F(x)-G(x)|\right\} .
$$

To test the null hypothesis that $F$ has a unimodal density Hartigan and Hartigan [5] proposed the statistic $D(F n)$, where $F n$ is the empirical distribution function of a random sample of size $n$. The distribution of $D(F n)$ is compared with the distribution of $D(F)$, where $F$ is the uniform distribution on $[0 ; 1]$. Although the dip test was originally reported as an unimodality measure, it is commonly used for computing the bimodality of distributions. Hartigan and Hartigan showed that the dip is asymptotically larger for the uniform distribution than for any other unimodal distribution. Therefore, histograms with high unimodality result in low scores of the dip test. High scores of the dip test correspond to highly bimodal histograms.

The algorithm for computing the dip test is published in [6]. Open source code used to compute the dip can be found inwww.cran.r-project.org/web/ packages/diptest/

\subsection{The Dynamics}

The dynamics 4, is a morphological measure associated to each regional extrema of an image. This measure is a powerful tool to quantify the saliency of a maximum or minimum. The dynamics of a regional minimum is defined as the minimum height we have to climb starting from this minimum to reach another one with strictly higher dynamics, the climb being the difference in altitude between the highest point of the path and the regional minimum under study. Formally: Let $M$ be a regional minimum of a function $f$. The dynamics of $M$ is defined as:

$$
\min \left\{\max _{s \in[0,1]}\{f(\gamma(s))-f(\gamma(0))\} \quad \mid \gamma:[0,1] \rightarrow \Re^{2}, f(\gamma(1))<f(\gamma(0)), \gamma(0) \in M\right\}
$$

where $\gamma$ is a path linking two points.

Note that the dynamics are not defined for the global minimum of the image, as there is no pixel with strictly lower altitude. However, in morphological image processing is assumed that the image $f$ has a global minimum on its boundary. This makes possible the valuation of the global minimum inside the domain of definition of $f$.

Grimaud [4],presents dynamics of regional minima instead of maxima. However, we need the dynamics of the maxima of the histogram to compute the bimodality. Dynamics of a regional maximum $M$ is the minimum height that we should fall, to reach another regional maximum higher than $M$.

\section{Computing the HOG Bimodality}

The algorithm for extracting the dynamics is based on the watershed algorithm proposed by Vincent and Soille [15. It consists in flooding from the minima, 
level by level, until water from a catchment basin meets water from another one. The meeting point between two basins is a saddle point, and this is the point where we can compute the dynamics of one of the two basins: the basin with the lowest value floods the other one, and the dynamics of the basin with the lowest minimum is equal to the grey-value of the saddle point minus the grey-value of the minima.

Organizing the image as a set of regions enables a faster implementation of dynamics. In this sense, the Component Tree [7, 9] is a structure formed by the decomposition of a grey-level image by thresholds, and very useful for dynamics determination [1]. It is especially interesting because it requires only the adjacency definition for its construction, and there exist several quasi-linear time algorithms for constructing it [10].

Max-tree refers to the algorithm introduced by Salembier 12 for an efficient implementation of the component tree. Silva and Lotufo describe in [14 an efficient computation of the dynamics, based on the incremental determination of attributes from the max-tree construction, in linear time.

We have used the implementation presented in [14] (Available from http://parati.dca.fee.unicamp.br/adesso/), and modified the original algorithm to make it able to work with cyclic histograms. As mentioned earlier, the dynamics of the absolute maximum of an image is computed considering that the image boundary has a virtual higher value. Since we are dealing with cyclic histograms, we cannot make this assumption because a circular structure has no boundaries. Thus, the global maximum of the histogram will be always considered the maximum with highest dynamics, and quantified with its height.

The other variation introduced to the original algorithm also concerns the circularity of HOGs. We have modified the original algorithm in order to avoid the zero crossing discontinuity. We have considered the circularity of the histogram in the flooding process, and taken into account that a saddle point can split two domes whose maxima are at both sides of the histogram origin. The zero crossing could also split a single dome in two, giving as a result two regional maxima, one at each side of the histogram origin. Due to the nature of the flooding process, this modification of the algorithm does not translate into any additional computational cost.

Once we have the values of the two highest dynamics, $d y n_{1}$ and $d y n_{2}$, the bimodality of the histogram is computed using equation 3

$$
B M=\frac{d y n_{1} \cdot d y n_{2}}{\sqrt{d y n_{1}^{2}+d y n_{2}^{2}}}
$$

The product of the dynamics is divided by the Euclidean norm for normalization purposes.

\section{Results}

We have computed the bimodality of a set of histograms, using both the dip test and our method based on dynamics. The objective is to establish a comparison 


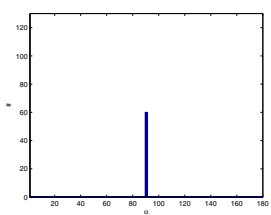

(a) $h_{1}$

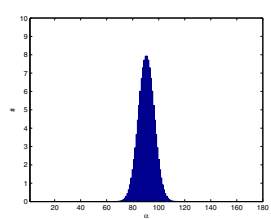

(e) smoothed $h_{1}$

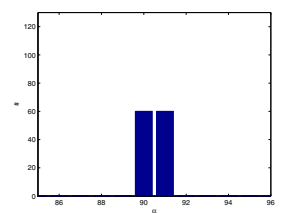

(b) detail of $h_{1}$

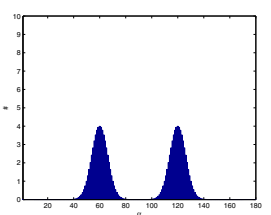

(f) smoothed $h_{2}$

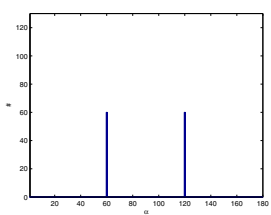

(c) $h_{2}$

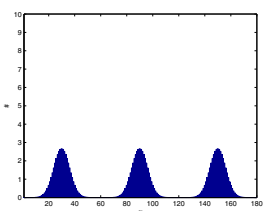

(g) smoothed $h_{3}$

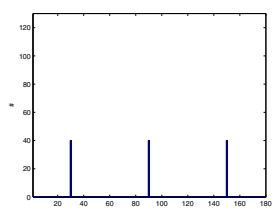

(d) $h_{3}$

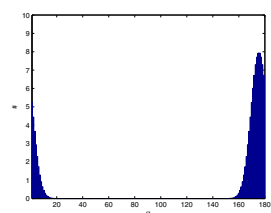

(h) shifted $h_{1}$

Fig. 3. Test histograms

between the responses of both algorithms. First we use synthetic histograms specially designed to detect anomalies in the bimodality measures. Next, we use the HOGs extracted from real images with the objective of evaluating the bimodality tests for non-ideal histograms.

$h_{1}$ is an unimodal histogram consisting of two non-empty neighbour bins. It is very usual when constructing HOGs, that the orientation of a single straight line count into two neighbour bins due to discretization. $h_{1}$ is a synthetic model of this situation. $h_{2}$ and $h_{3}$ are pure bimodal and trimodal histograms respectively. All histograms have the same amount of samples (120) and bins (180).

The first experiment is the response to histogram smoothing. In real images, the gradient direction, computed by means of convolution masks, is not always accurate. The measure of orientation is affected by blurring and lack of contrast of the image. This inaccuracy causes that HOG neighboring bins share samples from pixels with similar orientations, giving the typical modal shaped histogram. For this purpose we have smoothed the synthetic histograms $h_{1}, h_{2}$ and $h_{3}$, shown in figure 3, with a Gaussian filter with an increasing standard deviation.

Figure 4 shows the responses of bimodality tests for the 3 sets of filtered histograms. It should be noticed that the dip test gives a higher bimodality score to $h_{3}$ than to $h_{2}$ for high smoothing levels, while using our test, the bimodality of $h_{2}$ is always higher than $h_{3}$ for any given value of $\sigma$. But the main drawback of the dip test is that it gives the highest bimodality score $(0,25)$ to $h_{1}$. For descriptors such as HOGs, $h_{1}$ should always be considered as a pure unimodal histogram. Using our measure, $h_{1}$ gets the minimum bimodality score for all smoothing levels.

The second experiment is shift invariance. For a robust keypoint detection, rotational invariance is mandatory. Rotations of the image translate into cyclic shifts of the HOG. We have shifted the histograms shown in figures $3 \mathrm{e} 3 \mathrm{~g}$ by all possible values from 1 to 180 . The bimodality tests for the three sets of 180 


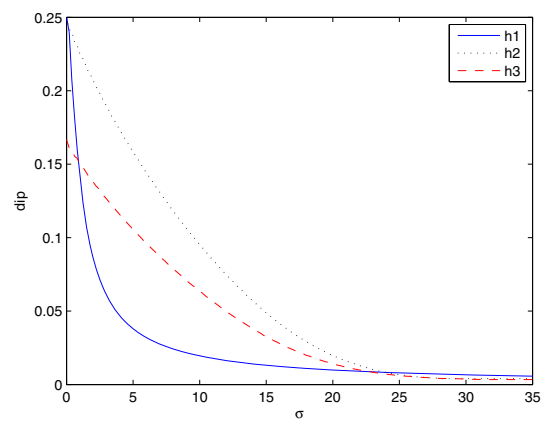

(a) dip test

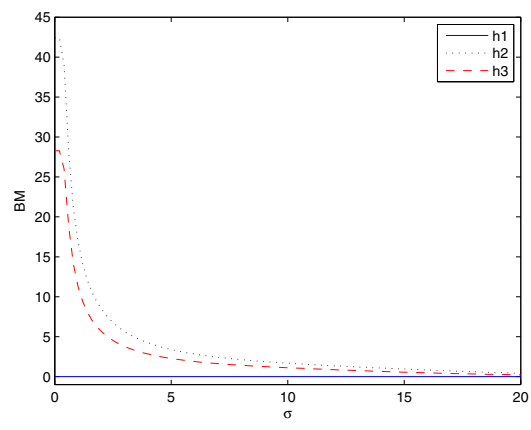

(b) based on dynamics

Fig. 4. Bimodality measures of smoothed histograms

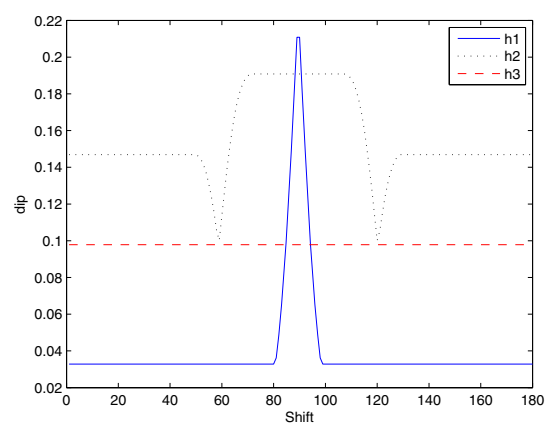

(a) dip test

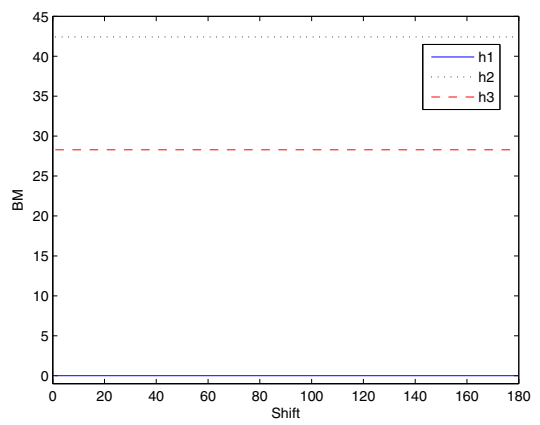

(b) based on dynamics

Fig. 5. Bimodality measures of shifted histograms

histograms have been computed, and the results are shown in figure 5, Due to the fact that the algorithm presented works with cyclic vectors, our bimodality measure remains constant for all possible shifts of every histogram. On the other hand, figure 5 a shows the effects of the zero crossing in the dip test. An unimodal histogram such as $h_{1}$, gets a high bimodality score when the mode is splitted in two parts at both sides of the histogram origin (like the one shown in figure 3h). A variation of the dip test for dealing with cyclic histograms is not an easy task, and we are not aware of any published implementation. However, a partial solution for using the dip algorithm with cyclic vectors, is to shift the histogram up to the global minimum before computing the dip test, but this solution doesn't work when the HOG has several global minima.

Finally, the third experiment is a comparison of the repeatability of the keypoints detected with both bimodality measures. 


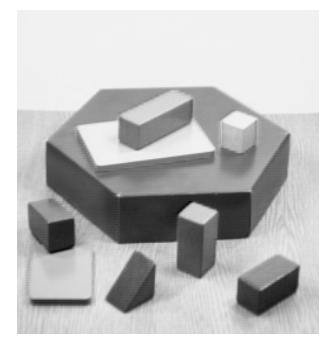

(a) Blocks image

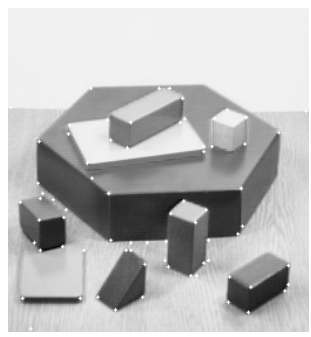

(b) Keypoints

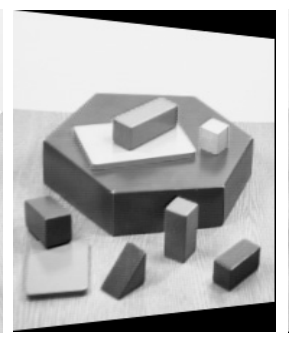

(c) $45^{\circ}$ transform

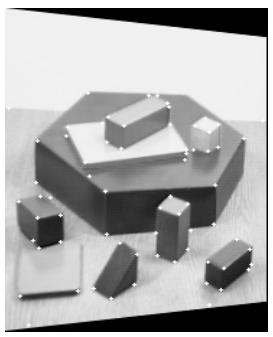

(d) Keypoints

Fig. 6. Test image, a projective transform, and keypoints detected

Using the integral histogram [11, a HOG is computed for every pixel of the image within a cell of $21 \times 21$ pixels. We construct the bimodality images (like the one shown in figure $2 \mathrm{~b}$ ) assigning the bimodality value of each histogram to the center pixel of each cell. Peaks of the bimodality image are candidates for being keypoints. We select the keypoints locating the relative maxima of the bimodality image. The bimodality scores make possible to establish a hierarchy of keypoints based on their saliency.

In order to test the robustness of the keypoints detected, we change the image point of view with a projective transform (see figure 6). Then, the whole process is repeated for both bimodality tests, computing new bimodality images and extracting the keypoints of the transformed images. Repeatability [13 is the standard measure for testing the robustness of a keypoint detector to image transforms. The repeatability rate, $r(I, \psi(I))$, is defined as the number of points present in both the original and the transformed image, with respect to the total number of detected points. Keypoints that are not present within a certain neighborhood in both images, decrease the repeatability measure. We have computed the repeatability $r\left(\psi_{\alpha}(\operatorname{Keyp}(I)), \operatorname{Keyp}\left(\psi_{\alpha}(I)\right)\right)$ for all projective transforms $\psi_{\alpha}$ from $\alpha=0^{\circ}$ to $\alpha=70^{\circ}$. Keyp $(I)$ is an image containing the keypoints of image $I$. It is computed for both bimodality measures selecting the most salient maxima of the bimodality images. As it could be expected from previous experiments, we have obtained a higher repeatability using the bimodality measure based on dynamics than using the dip test. Concretely, using the dip test we obtain a mean repeatability $\bar{r}=66.2 \%$, while using our measure the mean repeatability is $\bar{r}=78,8 \%$.

\section{Conclusions}

The dip test of bimodality presents some drawbacks that create big problems when computing the bimodality of HOGs: it does not deal with cyclic histograms, unimodal histograms consisting of two neighboring bins are considered bimodal, and it does not preserve the order of the scores when the smoothing level is increased. 
We have presented a new algorithm for computing the bimodality of an histogram based on the dynamics of its domes. This algorithm overcomes the drawbacks of the dip test. The keypoints detected in real images by means of HOG bimodality, have shown to have a higher repeatibility using our measure.

Acknowledgments. This research was partially supported by Consolider Ingenio 2010, project (CSD2007-00018) and CICYT project DPI2010-17112.

\section{References}

1. Bertrand, G.: On the dynamics. Image Vision Comput. 25(4), 447-454 (2007)

2. Cataño, M.A., Climent, J.: Keypoint detection based on the unimodality test of hOGs. In: Bebis, G. (ed.) ISVC 2012, Part I. LNCS, vol. 7431, pp. 189-198. Springer, Heidelberg (2012)

3. Dalal, N., Triggs, B.: Histograms of Oriented Gradients for Human Detection. In: IEEE Conference on CVPR, vol. 1, pp. 886-893 (2005)

4. Grimaud, M.: New measure of contrast: the dynamics. Image Algebra and Morphological Image Processing III 1769(1), 292-305 (1992)

5. Hartigan, J.A., Hartigan, P.M.: The Dip Test of Unimodality. Ann. Stat. 13(1), 70-84 (1985)

6. Hartigan, P.M.: Algorithm as 217: Computation of the dip statistic to test for unimodality. J. Roy. Stat. Soc. C-App. 34(3), 320-325 (1985)

7. Jones, R.: Connected filtering and segmentation using component trees. Comput. Vis. Image Und. 75(3), 215-228 (1999)

8. Lowe, D.G.: Distinctive Image Features from Scale-Invariant Keypoints. International Journal of Computer Vision 60(2), 91-110 (2004)

9. Mattes, J., Richard, M., Demongeot, J.: Tree Representation for Image Matching and Object Recognition. In: Bertrand, G., Couprie, M., Perroton, L. (eds.) DGCI 1999. LNCS, vol. 1568, pp. 298-312. Springer, Heidelberg (1999)

10. Najman, L., Couprie, M.: Building the component tree in quasi-linear time. IEEE T. Image Process. 15(11), 3531-3539 (2006)

11. Porikli, F.: Integral histogram: A fast way to extract histograms in cartesian spaces. In: IEEE Computer Vision and Pattern Recognition (CVPR), pp. 829-836 (2005)

12. Salembier, P., Oliveras, A., Garrido, L.: Antiextensive connected operators for image and sequence processing. IEEE T. Image Process. 7(4), 555-570 (1998)

13. Schmid, C., Mohr, R., Bauckhage, C.: Comparing and Evaluating Interest Points. In: ICCV, pp. 230-235 (1998)

14. Silva, A.G., de Alencar Lotufo, R.: Efficient computation of new extinction values from extended component tree. Pattern Recogn. Lett. 32(1), 79-90 (2011)

15. Vincent, L., Soille, P.: Watersheds in digital spaces: An efficient algorithm based on immersion simulations. IEEE T. Pattern Anal. 13(6), 583-598 (1991) 